\title{
Melanocortin 4 Receptor Mediates Neuropathic Pain Through p38MAPK in Spinal Cord
}

\author{
Haichen Chu, Jiangling Xia, Hongmei Xu, Zhao Yang, Jie Gao, Shihai Liu
}

\begin{abstract}
Background: Neuropathic pain is characterised by spontaneous ongoing or shooting pain and evoked amplified pain responses after noxious or non-noxious stimuli. Neuropathic pain develops as a result of lesions or disease affecting the somatosensory nervous system either in the periphery or centrally. Melanocortin 4 receptor (MC4R) plays an important role in the initiation of neuropathic pain but the underlying mechanisms are still unclear. Methods: Adult male Wistar rats were given chronic constriction injury (CCI) or sham operations. Part of CCI rats were intrathecally treated with HS014 (MC4R antagonist) or SB203580 (p38MAPK inhibitor). On the third, seventh and fourteenth day, the thermal threshold of operated paws was tested. In addition, the MC4R or phosphorylated p38MAPK (p-p38MAPK) levels of lumbar spinal cord were tested with ELISA (enzyme-linked immunosorbent assay), western blot and immunohistochemistry. Results: Here we demonstrate that (1) both HS014 and SB203580 reduced CCI reduced hyperalgesia (2) p-p38MAPK was increased after CCI with a time course parallel to that of the MC4R change, (3) The p38 activation was prevented by blocking MC4R with an antagonist HS014, but MC4R-IR was not prevented by SB203580. (4) MC4R and pp38MAPK were located in the same cells. Conclusion: The mechanisms of neuropathic pain mediated by MC4R is related to the inhibition of p38MAPK activation. P38MAPK may be a downstream of MC4R.
\end{abstract}

RÉSUMÉ: Médiation de la douleur neuropathique par le récepteur de la mélanocortine 4 par l'entremise de p38MAPK dans la moelle épinière. Contexte : La douleur neuropathique est caractérisée par une douleur spontanée persistante ou lancinante et des réponses douloureuses amplifiées évoquées suite à des stimuli, qu'ils soient nocifs ou non. La douleur neuropathique apparaît suite à des lésions ou à une maladie qui touche le système nerveux somatosensitif périphérique ou central. Le récepteur de la mélanocortine 4 (MC4R) joue un rôle important dans la genèse de la douleur neuropathique. Les mécanismes sous-jacents demeurent cependant mal connus. Méthode : Des rats Wistar mâles adultes ont subi une lésion par constriction chronique (LCC) ou une intervention factice. Une partie des rats ayant subi une LCC ont été traités par le HS014 (un antagoniste du MC4R) par voie intrathécale ou par le SB203580 (un inhibiteur de p38MAPK). Les seuils thermiques au niveau des pattes qui avaient subi l'intervention ont été évalués le 3e, le 7e et le 14e jour après l'intervention et le MC4R ou le p38MAPK phosphorylé (p-p39MAPK) au niveau de la moelle épinière lombaire ont été mesurés par la méthode de dosage ELISA, buvardage western et immunohistochimie. Résultats : Nous avons fait les observations suivantes : tant le HS014 que le SB203580 diminuaient l'hyperalgésie due à la LCC; le p-p38MAPK était augmenté après la LCC et son évolution dans le temps était parallèle au changement du MC4R ; le fait de bloquer le MC4R avec un antagoniste comme le HS014 prévenait l'activation du p38, mais le SB203580 ne prévenait pas l'immunoréaction avec le MC4R ; et le MC4R et le p-p38MAPK étaient présents dans les mêmes cellules. Conclusion : Le mécanisme de la douleur neuropathique dont la médiation se fait par le MC4R est en lien avec l'inhibition de l'activation par le p38MAPK et le p38MAPK pourrait se situer en aval du MC4R.

Can J Neurol Sci. 2012; 39: 458-464

Peripheral nerve injury often results in neuropathic pain associated with hyperalgesia and allodynia ${ }^{1}$. Several lines of evidence suggest that melanocortin 4 receptor (MC4R) plays an important role in the initiation of neuropathic pain. MC4R is activated after nerve injury. Intraplantar injection of MC4 receptor antagonists can abolish chronic constriction injury (CCI)-induced allodynia and hyperalgesia ${ }^{2}$. It suggests that MC4R is involved in the transduction of neuropathic pain. Recently MC4R was found in dorsal root ganglia (DRG) where nerve injury can affect its expression ${ }^{3}$, but the underlying mechanisms are still unclear. P38MAPK was improved to play an important role in the development and maintenance of nerve injury, inflammation and incision pain ${ }^{4-9}$. Intrathecal inhibitor of p38 has been shown to attenuate neuropathic pain in different animal models $\mathrm{s}^{4,10}$. Furthermore MC4R can activate MAPK in vitro $^{11,12}$. Therefore, we hypothesized that activation of the $\mathrm{p} 38$ cascade may represent a route by which MC4R could trigger changes in transcription as well as post-translational

From the Department of Anesthesiology (HC, JX, HX, JG), Qingdao Institute for Drug Control (ZY), Central Laboratory (SL), Affiliate Hospital of Qingdao University Medical College, Qingdao, Shandong Province, China.

Received June 28, 2011. Final Revisions Submitted February 7, 2012. Correspondence to: Haichen Chu, Department of Anesthesiology, Affiliate Hospital of Qingdao University Medical College, Qingdao 266003, Shandong Province, China. Email: haichen0312@yahoo.com.cn 
modifications of target proteins in the spinal cord that are correlated with the development of pain after nerve injury.

In the present study, we investigated whether the inhibition of MC4R or p38 could block the development of hyperalgesia after $\mathrm{CCI}$ and we looked at the time course of p38 activation in spinal cord after CCI. In addition to examining covariance among the susceptibility of CCI-associated hyperalgesia to MC4R antagonism, p38 antagonism, MC4R activation and p38 activation, we determined whether inhibition of MC4R blocked the activation of $\mathrm{p} 38$, If CCI-induced increases in spinal pp38MAPK levels are not antagonized by spinal HS014, but thermal hyperalgesia were antagonized partly, we will conclude that $\mathrm{p} 38$ is phosphorylated by different pharmacological triggers in the spinal cord. This would imply that the increased spinal MC4R seen following nerve injury is exclusively down and not upstream of p38 activation, thus for the first time, demonstrating a causal link between nerve injury, MC4R, and p38MAPK.

\section{Materials ANd Methods}

1.Animals. Experiments were performed on male Wistar rats weighing $300 \pm 20 \mathrm{~g}$ at the time of surgery. Animals were housed individually in cages lined with sawdust. Standard laboratory rodent chow and water were available. The rats were maintained under a 12 hour light/dark cycle (lights were turned on at 8:00 a.m.). All behavioral experiments were conducted during the light phase of the cycle, between 9:00 a.m. and 2:00 p.m.

2. Surgical preparations. Chronic constriction injury (CCI) was produced by loosely tying four ligatures (4/0 silk Shanghai Jinhuan Medical Co. Ltd) around the sciatic nerve under chloral hydrate anesthesia (300 mg/kg; i.p.). The biceps femoris and the gluteus superficial were separated, and the right sciatic nerve was exposed. The ligatures (4/0 silk) were tied loosely around the nerve, distal to the sciatic notch, with $1 \mathrm{~mm}$ spacing until a brief twitch in the respective hind limb was observed as previously described ${ }^{13}$. After surgery, all rats developed longlasting neuropathic pain symptoms measured by heat sensitivity. Sciatic nerve was exposed but not ligated in sham operated animals.

3. Drug delivery. In all the animals, 7-mm-long polyethylene (PE10) catheters were implanted into the intrathecal space ${ }^{14}$. Catheters were connected to a $25 \mu$ l Hamilton syringe. MC4R antagonists (HS014 H2396 SIGMA) or p38MAPK antagonists (SB203580 S8037 SIGMA) were given daily for seven days following CCI, by means of a 25 ul Hamilton syringe, followed by a saline flush $(12 \mu \mathrm{l})$. HS014 dissolved in saline and $5 \mu \mathrm{g} / \mathrm{d}$. SB203580 dissolved in DMSO and used $10 \mathrm{mg} / \mathrm{d}$. As a control, $\mathrm{CCI}$ and sham group animals were treated with $10 \mu \mathrm{l}$ saline for seven days instead. We analyzed samples taken from rats on 3d,7d, 14d after CCI.

4. Behavior testing. For assessment of thermal hyperalgesia, Rats were tested for paw withdrawal latency in response to noxious thermal stimuli using an hot plate measuring pain instrument (Baishi Electronic Medical Instruments Company). When the temperature reached $55^{\circ} \mathrm{C}$ animals were placed on it. Then we count the time they lifted their operated paw. This was repeated three times with a ten minute interval between each testing.

5. Immunohistochemistry. Three, seven and 14 days after $\mathrm{CCI}$ or sham surgery, the rats $(\mathrm{n}=4$ per group) were deeply anesthetized with chloral hydrate $(400 \mathrm{mg} / \mathrm{kg}$, ip) and transcardially perfused with $60 \mathrm{~mL}$ of saline $(0.9 \% \mathrm{NaCl})$, followed by $300 \mathrm{ml}$ of $4 \%(\mathrm{w} / \mathrm{v})$ paraformaldehyde with $4 \%$ paraformaldehyde in $0.1 \mathrm{M}$ phosphate-buffered solution $(\mathrm{pH}$ 6.9). The lumbar spinal cord was removed and post-fixed in the same fixatives. The tissues were embedded in paraffin and then cut into $3 \mu \mathrm{m}$ thick sections using a Micro cryostat. Free-floating spinal cord sections were incubated in $37^{\circ} \mathrm{C}$ water for two hours with rabbit polyclonal antibodies against the $\mathrm{MC} 4$ receptor (1:300; Abcam, Cambridge, UK) or p-p38MAPK (1:200 bs0636R Beijing Biosynthesis Biotechnology Co., Ltd). The sections were then incubated for half an hour at $37^{\circ} \mathrm{C}$ water with goat anti-rabbit secondary antibodies (Zhongshan Golden Bridge Biotechnology Co. Ltd). Finally, the sections were washed and stained with 3', 3'- diaminobenzidine tetra hydrochloride (DAB) for five minutes. Then, the spinal cord

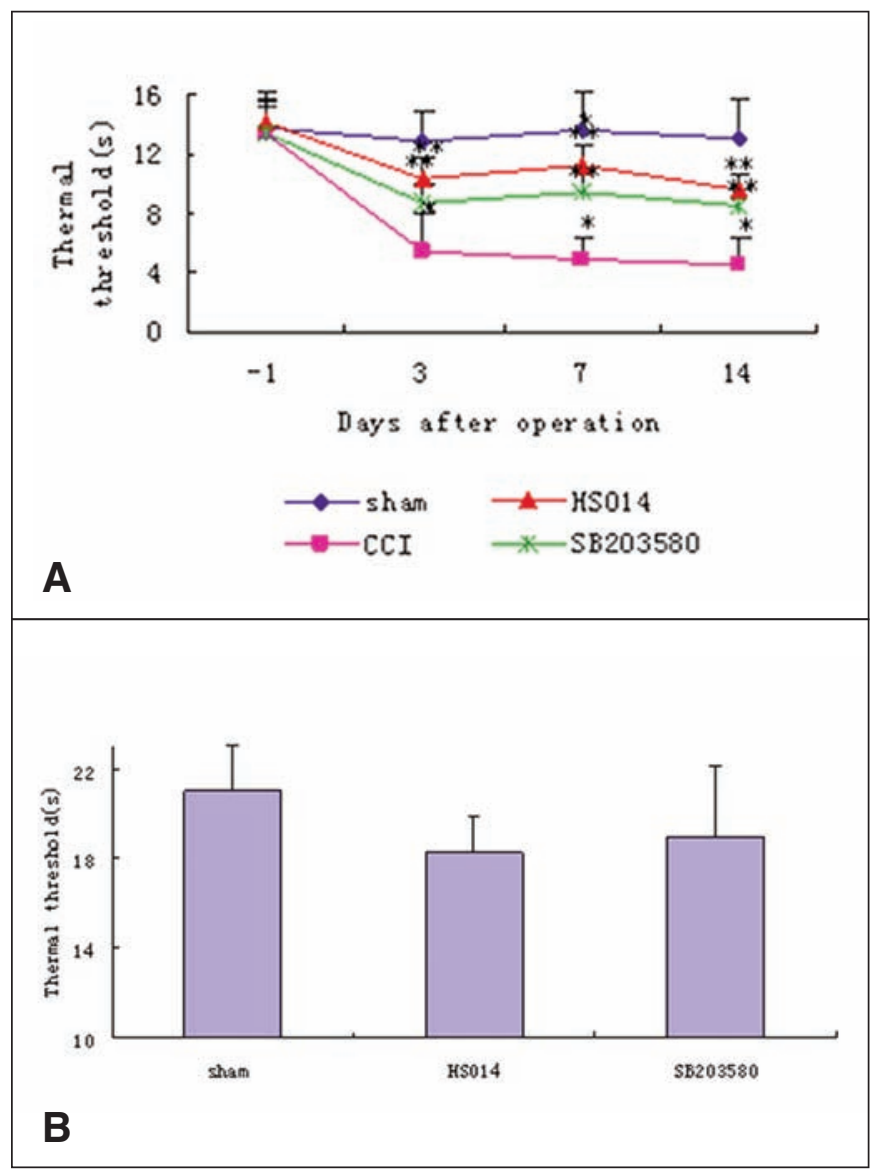

Figure 1: Development of chronic constriction injury (CCI)-induced hyperalgesia with or without treatment after injury. (A) Hyperalgesia were measured $1 d$ before and 3, 7 and 14 days after operation of different groups.(B) showed the effect of HSO14 and SB203580 on hyperalgesia in sham operated rats. The time relating to operation (days) is plotted along the $X$-axis and the time (seconds) animals lift up their operated paws is plotted on the $Y$-axis. $*<0.01$ versus sham group. $* *<0.05$ versus $C C I$ group. 
sections were washed in tap water, the floating spinal cord sections were mounted on gelatin-coated slides, and all sections were dehydrated in alcohol, cleared in xylene, and mounted in dibutylphthalate polystyrene xylene (DPX; Merck, Darmstadt, Germany). To demonstrate the specificity of the staining, the omission of either the primary antibody, the secondary antibody or the avidin-biotin complex was performed (data not shown). For double immunofluorescence, spinal sections were incubated with a mixture of polyclonal MC4R antibody (1:100, Santa) and polyclonal p-p38MAPK antibody (1:400, Cell signaling) overnight at $4^{\circ} \mathrm{C}$, followed by a mixture of FITC- and TITRCconjugated secondary antibodies (1:50, Zhongshan Golden Bridge Biotechnology Co. Ltd) for one hour at $37^{\circ} \mathrm{C}$ water. The stained sections were examined with a Leica microscope, and images were captured with a CCD Spot camera.
6. ELISA and Western blot were used for p-p38MAPK analysis. The animals were anesthetized with chloral hydrate, and euthanized with an intracardiac injection of saturated potassium chloride. The lumbar spinal cord was exposed by laminectomy, and approximate $2 \mathrm{~cm}$ length of tissue around L45 segment was excised and placed into a Petri dish containing dry-ice-cold homogenizing buffer. Tissue from all of animals was processed at the same time. The lumbar spinal cord were weighed and homogenized in cold extraction Trisbuffered saline (pH 8.0) containing 1\% NP-40, 10\% glycerol, $0.5 \mathrm{mM}$ sodium metavanadate, $1 \mathrm{mM}$ phenylmethylsulfonyl fluoride, $10 \mathrm{mg} / \mathrm{ml}$ aprotinin, and $1 \mathrm{mg} / \mathrm{ml}$ leupeptin. The lysates were centrifuged for 15 minutes, and a commercially available enzyme-linked immunosorbent assay (ELISA) kit (CK-E90668R R \&D) was used to detect p-p38MAPK in the supernatants. P-p38MAPK levels in the lumbar spinal cord were normalized to the amount

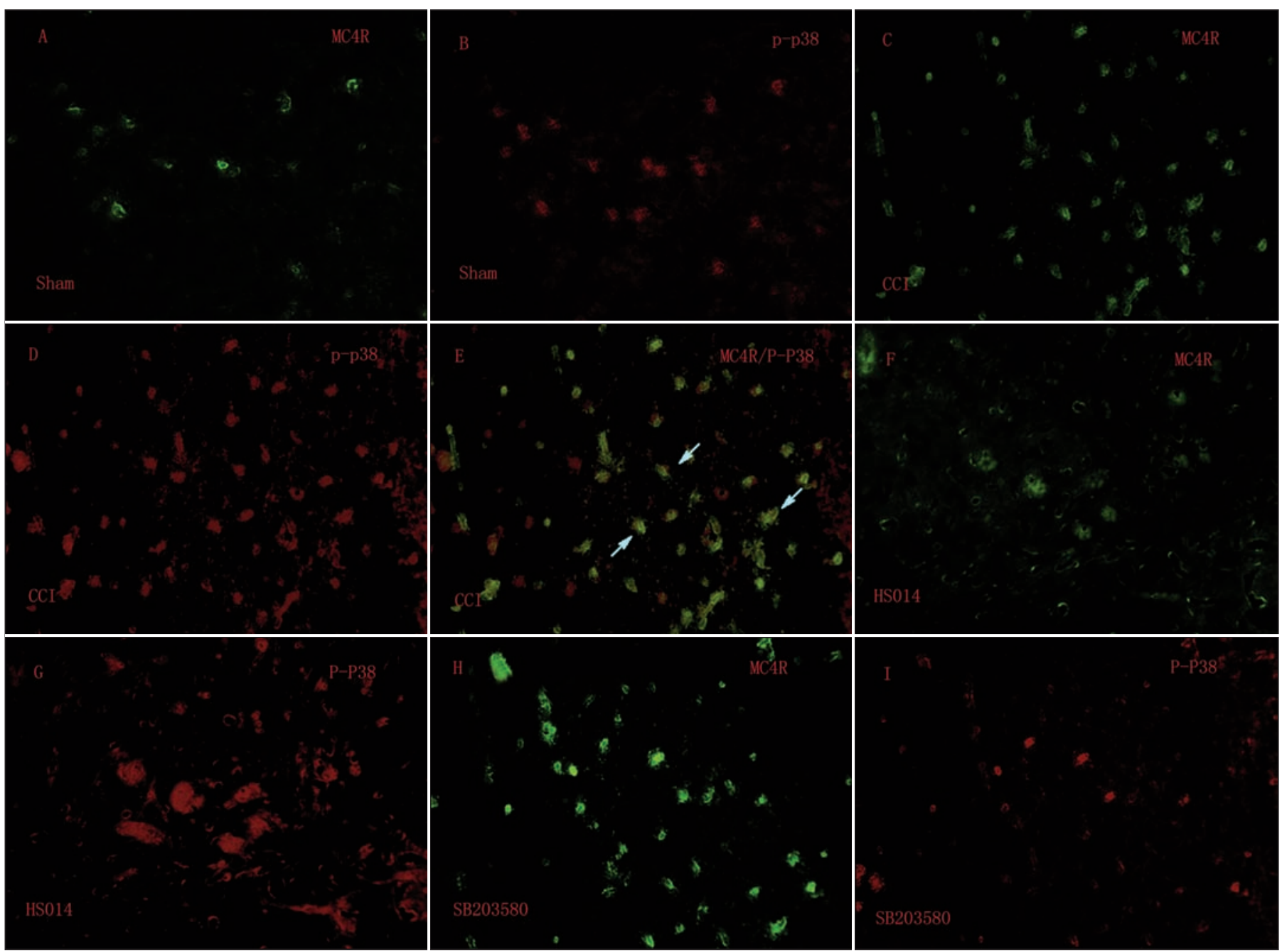

Figure 2: Effect of drug treatment after chronic constriction injury to the sciatic nerve on MC4R immunoreactivity(IR) and p-p38MAPK in the rat spinal cord. Shown are representative images of MC4R and p-p38MAPK in the ipsilaterative spinal cord in sham operated animals $(A, B), C C I$ group $(C, D)$, HSO14 group $(F, G)$ and SB203580 (H,I) group. Both MC4R and p-p38 significantly increased after CCI. E is a picture of both MC4R and p-p38 after CCI, double immunofluorescence shows that MC4R is colocalized with p-p38MAPK (arrows). 
of total protein. Protein samples were separated on SDS-PAGE gel $(10 \%)$ and transferred to PVDF Wlters (Millipore). The blots were blocked with $5 \%$ milk in PBS with $0.1 \%$ Tween-20 for one hour at room temperature (RT) and incubated overnight at $4{ }^{\circ} \mathrm{C}$ with polyclonal anti-MC4R antibody $(1: 1000$, Santa, antirabbit), p38MAPK antibody (1:2000, Cell Signaling, antimouse) or p-p38MAPK antibody (1:2000, Cell Signaling, antimouse). The blots were then incubated for one hour at RT with HRP-conjugated secondary antibody (Zhongshan Golden Bridge Biotechnology Co. Ltd, 1:3000), developed in ECL solution (NEN) for one minute, and exposed onto hyperflims for 1-30 minutes.

7. Statistical analysis. Immunohistochemical data are presented as the mean standard error of the mean (SEM). Differences in the percentage of phosphorylated p38MAPK and MC4 receptor-expressing neurons in the spinal cord between sham-operated and neuropathic rats were tested using one-way analysis of variance (ANOVA) followed by the Bonferroni test. Differences were considered significant if $\mathrm{p}<0.05$.

\section{RESULTS}

Nerve injury results in thermal hyperalgesia, as demonstrated by significantly shorter latency of paw lift from the heat plate (from 3 to 14 days after CCI) $(\mathrm{p}<0.01)$. The average time of sham group is $13.2 \pm 2.2 \mathrm{~s}$ and CCI shorted the time by $66 \%$ to $4.5 \pm 1.9 \mathrm{~s}$ on the fourteenth day after CCI. HS014 treatment at the moment of CCI attenuated thermal hyperalgesia significantly $(\mathrm{p}<0.05)$. After treatment for seven days the latency prolonged to $6.9 \pm 1.3 \mathrm{~s}$. We next investigated the effects of p38 inhibition in rats with CCI. Animals treated with intrathecal SB203580 displayed an inferior pattern to those with intrathecal MC4R antagonist. Treatment with the p38 antagonist reduced the CCIinduced hyperalgesia significantly $(\mathrm{p}<0.05)$ (Figure $1 \mathrm{~A})$, Cessation of drug treatment resulted in no loss of the antihyperalgesia effect. There are no significant differences in thermal hyperalgesia between with and without treatment of HS014 or SB203580. Treatment with HS014 or SB203580 had no effect on the hyperalgesia of sham operated rats (Figure 1B).

MC4R expression increased after nerve injury in the spinal cord ipsilateral to the CCI (Figure 2). Quantitative evaluation showed MC4 receptor-IR in $21.6 \pm 2.7 \%$ of spinal cord (Figure $3 \mathrm{~A})$. Seven days after CCI , $38.3 \pm 1.5 \%$ of spinal cord cells were MC4 receptor-positive, which represents a relatively significant increase of $16.7 \%$ on the injured side compared to the sham group ( $<<0.01$; Figure 3A).

P38MAPK phosphorylation, increased in number after nerve injury in spinal cord ipsilateral to the CCI (Figure 2D). Quantitative evaluation showed p-p38MAPK in $23 \pm 2.3 \%$ of spinal cord cells (Figure 4A). Seven days after CCI, $34 \pm 1.0 \%$ of spinal cord were p-p38MAPK positive, which reached the peak and represented a relatively significant increase of $11 \%$ on the injured side compared to the sham group ( $<<0.01$; Figure $4 \mathrm{~A})$.

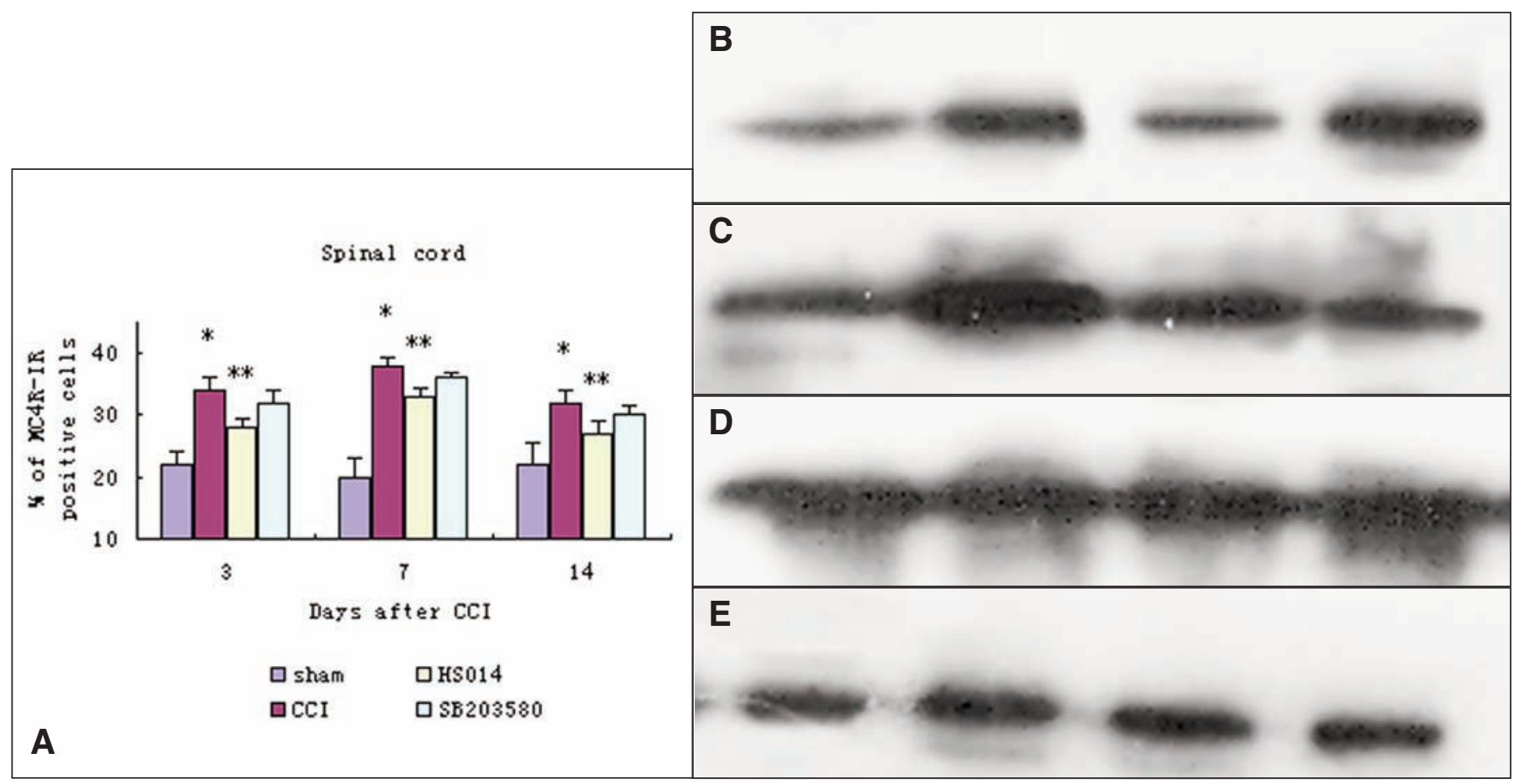

Figure 3: (A) Quantification of MC4R stained cells. The percentage of the immunostained MC4R cells increases following injury. The rate of positive $M C 4 R$ cells reaches the peak 7 days after CCI and lasting for 14 days. Intrathecal HSO14 decreases the MC4R-IR significantly. Values are presented as the mean SEM; $n=24$ per group. $* 0.01$ versus sham group $; * *<0.05$ versus CCI group. (B)Western blot shows expressions of MC4R (A), p-p38MAPK $(B), p 38 M A P K(C)$ and GAPDH $(D)$ in the spinal cord seven days after CCI in different groups. From the left they are sham group, CCI group, HSO14 group and SB203580 group. CCI induces an increase of both MC4R and p-p38MAPK, without changes of p38MAPK. 


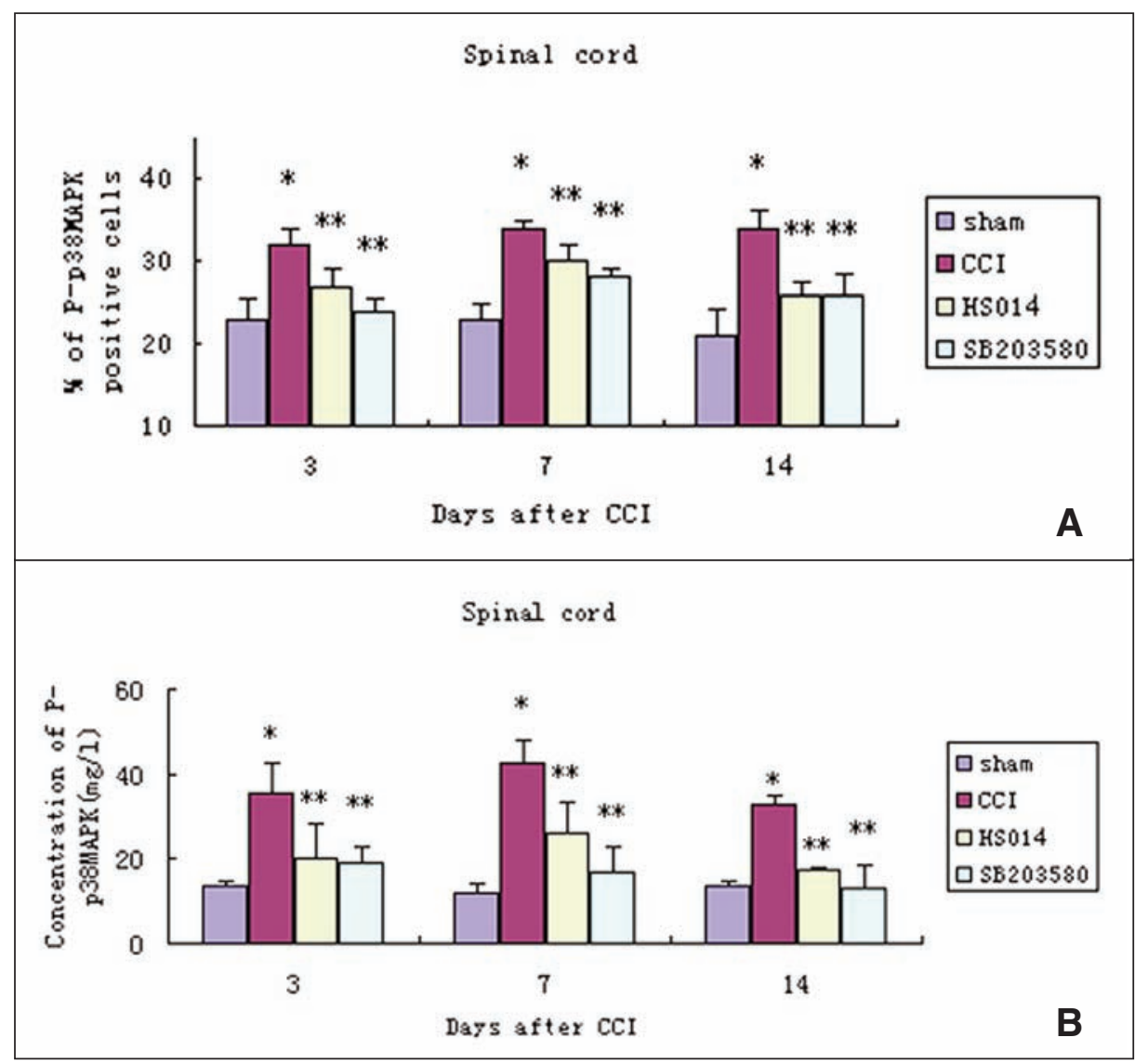

Figure 4: (A) Quantification of p-p38MAPK stained cells. the percentage of the p-p38MAPK cells increase following injury. CCI resulted in an increase in p-p38MAPK ipsilateral to injury. Intrathecal HSO14 or SB203580 decreased the p-p38MAPK significantly. Values are presented as the mean SEM; $n=24$ per group. Concentration of p-p38MAPK in each group of spinal cord detected by ELISA (B). CCI induced an increase of p-p38MAPK significantly. Treatment with HSO14 or SB203580 attenuate the concentration of p-p38MAPK in spinal cord. $* 0.01$ versus sham group; $* *<0.05$ versus CCI group.

ELISA tests show a statistically significantly increase of $\mathrm{p}$ p38MAPK in spinal cord after CCI $(\mathrm{P}<0.01)$, versus the shamoperated group(13.1 \pm 1.6$)$, CCI group peaks $(42.4 \pm 5.6)$ on the seventh day after CCI (Figure 4B), which is similar to the changes in MC4R.

To test whether MC4R plays a role in p38 activation after CCI, the MC4R antagonist HS014 $(5 \mu \mathrm{g} / \mathrm{d})$ was given at the time of CCI. The spinal lumbar enlargements were harvested $3 \mathrm{~d}, 7 \mathrm{~d}$ , $14 \mathrm{~d}$ after CCI and analyzed for p38 phosphorylation using ELISA and immunohistochemistry. As seen in Figure 4, CCI induced a significant increase in phosphor-p38 in spinal cord. Intrathecal injection with HS014 $(5 \mu \mathrm{g} / \mathrm{d})$ daily for seven days markedly inhibited the CCI-induced activation of p38 in spinal cord (reduced by 7\%) (Figure 4A). ELISA tests showed that concentration of P-p38MAPK in spinal cord is $17.4 \pm 0.8 \mathrm{mg} / 1$ after HS014 treatment (Figure 4B). Thermal hyperalgesia were also significantly attenuated by treatment with HS014 (Figure 1A). Double immunofluorescence also shown that MC4R and p38MAPK both located on the same cells (Figure 2E). As shown in Figure 3B, Western blot analysis confirmed the results. CCI induced high expression of MC4R and p-p38MPK. Both HS014 and SB203580 can decrease the expression of p-p38MAPK without changing the expression of total p38 protein, while SB203580 cannot inhibit the increase of MC4R after CCI.

\section{DISCUSSION}

The aim of the present experiment is to study the mechanism of MC4R in neuropathic pain, especially in the early stages after CCI. We behaviorally assessed the alleviation of hyperalgesia chronic pain symptoms after intrathecal administration of MC4 receptor antagonist and p38 inhibitor. We demonstrated an upregulation of p-p38MAPK in the dorsal horn of the lumbar spinal cord and MC4R antagonists could decrease the phosphorylation of p38 in CCI-induced neuropathic pain. The correlation between behavioral and biochemical changes suggests the possibility that blocking MC4 receptors that are activated by injury attenuate neuropathic pain through p38MAPK pathway.

Since MC4R was cloned in 1993 by degenerate PCR, it has attracted widespread attention. Extensive localization studies on 
rat showed that the MC4R is widely expressed in adult rat, including cortex, thalamus, hypothalamus, brainstem, spinal cord and epidermal melanocytes ${ }^{15-18}$. Many studies have established that MC4R plays an important role in regulating energy homeostasis, food intake, cardiovascular function and sexual function ${ }^{19}$. Recently MC4R was found in $\mathrm{DRG}^{3}$ and astrocytes $^{20,21}$, which are involved in the development and maintenance of neuropathic pain. Administration of HSO14 (MC4R antagonist) with morphine delays the development of tolerance and prevents withdrawal hyperalgesia ${ }^{22}$. After chronic constriction injury of sciatic nerve, MC4R in both DRG and spinal cord were increased. In parallel with these findings, antagonism of MC4R reduces allodynia and hyperalgesia $a^{2,23,24}$. The present data obtained using the CCI model support the hypothesis that MC4R plays a causal role in neuropathic pain.

P38MAPK both play an important role in the development and maintenance of neuropathic pain. Pretreatment with a p38 antagonist intrathecally (i.t) can prevent SNL (spinal nerve ligation $)^{25}$ and $\mathrm{CCI}^{26}$ induced allodynia. Treatment with a p38 blocker, SB203580, five days after CCI also reduced pain behavior $^{27}$. Activated p38 in DRG and spinal cord was transiently elevated after SNL and then recovered to basal levels ${ }^{25} . \mathrm{Xu}^{26}, \mathrm{Lee}^{28}$ and present data indicate that activated p38 in spinal cord elevated after CCI and sustained for 14 days and more. Differences among the various models seem to cause substantial differences in spinal p38 activation. Both MC4R and p38 are involved in the development and maintenance of neuropathic pain. Also immunofluorescence indicates MC4R and p-p38 in the spinal cord were both located in the same cells. This provides an anatomical base for their cascaded relationship. The present study showed that CCI induced an elevation of pp38 without changes of $\mathrm{p} 38$, indicating that the increase in phosphor-p38 after nerve injury is caused by increased phosphorylation rather by elevated substrate.

The therapeutic window that we observed for HS014 corresponded to the activation of phosphor-p38 in the spinal cord. Intrathecal administration of SB203580, a p38 antagonist, was also effective when started immediately after the injury, thus both MC4R and p38 activation seem to be necessary for the initiation of hyperalgesia. Treatment with HS014 or SB203580 had no effect on the hyperalgesia of sham operated rats. That is to say HS014 and SB203580 could not elevate the thermal threshold of rats without neuropathic pain. HS014 and SB203580 relieve CCI induced neuropathic pain through acting on high levels of MC4R or p-p38MAPK. A perplexing fact is that phosphor-38 was not returned to basal levels in spinal cord 14 days after CCI, while interruption of either antagonist after seven days of therapy results in no loss of the anti-hyperalgesia effect. After CCI, a peripheral generator likely develops that drives central sensitization and pain behavior through a MC4Rp38 linkage. Nerve conduction blockade with bupivacaine does not reverse p38 activation when applied after sciatic nerve injury. It is suggested that after initiation, microglia activation could be self-maintained given that multiple mediators released by microglia can also feed back to microglia via an autocrine or paracrine pathway ${ }^{29}$. Future experiments will examine this possibility indepth.

Our data suggest a possible sequence of events related to the expression of p38 and MC4R in CCI rats. Furthermore MC4R- mediated inhibition of apoptosis in immortalized hypothalamic neurons is via mitogen-activated protein kinase $\mathrm{s}^{30}$. One critical site involved in this process is the spinal cord ipsilateral to nerve injury, because after CCI MC4R and p-p38MAPK of spinal cord are increased significantly. Several lines of evidence support a role of p38 in the cascade with MC4R: (1) p-p38MAPK was increased after CCI with a time course parallel to that of the MC4R change. Both ELISA and immunohistochemistry results demonstrate that. (2) The p38 activation was prevented by blocking MC4R with an antagonist HS014. (3) The MC4R expression was not diminished by p38 antagonist (SB203580). (4) MC4R and p-p38MAPK are located in the same cells. These findings strongly support the hypothesis that HS014 blocking neuropathic pain by inhibiting the activation of $\mathrm{p} 38$. MC4R and p38 linked at one point in spinal cord. MC4R trigger p38 activation. However, these do not exclude the possibility that additional pathways that MC4R mediates to prevent neuropathic pain $^{22,31}$. At the same time treatment with HS014 cannot prevent pain behavior completely. There maybe other pathways CCIinduced neuropathic pain can mediate which needs to be further investigated.

In summary, treatment with a MC4R antagonist or a p38 antagonist at the moment of CCI attenuates hyperalgesia induced by CCI. Antagonism of MC4R not only reduces pain behavior but also blocks activation of p38. After CCI, there is a longlasting increase in phosphor-p38 in spinal cord, which peaked on the seventh day. MC4R also expresses in the same cells with pp38MAPK. Together, these data implicate the MC4R-p38 signal transduction cascade in the spinal cord as a significant participant in the generation of hyperalgesia after nerve injury.

\section{ACKNOWLEDGEMENTS}

This work was supported by the Natural Science Foundation of Shandong Province, No.Y2006C11*. 


\section{REFERENCES}

1. Tsuda M, Mizokashi A, Shigemoto-Mogami Y, et a1. Activation of P38 mitogen activated protein kinase in spinal hyperactive microglia contributes to pain hypersensitivity following peripheral nerve injury. Glia. 2004;45:89-95.

2. Starowicz K, Mousa SA, Obara1 I, et al. Peripheral antinociceptive effects of MC4receptor antagonists in a rat model of neuropathicpain - a biochemical and behavioral study. J Pharmacol rep. 2009;61:1086-95.

3. Starowicz K, Bilecki W, Sieja A, Przewlocka B, Przewlocki R. Melanocortin 4 receptor is expressed in the dorsal root ganglions and down-regulated in neuropathic rats. Neurosc Lett. 2004;358: 79-82.

4. Wen YR, Suter MR, Ji RR, et al. Activation of p38 mitogenactivated protein kinase in spinal microglia contributes to incision-induced mechanical allodynia. Anesthesiology. 2009; 110:155-65.

5. Milligan ED, Twining C, Chacur $\mathrm{M}$, et al. Spinal glia and proinflammatory cytokines mediate mirror-image neuropathic pain in rats. J Neurosci. 2003;23:1026-40.

6. Ji R, Samad T, Jin S, Schmoll R, Woolf C. p38 MAPK activation by NGF in primary sensory neurons after inflammation increases TRPV1 levels and maintains heat hyperalgesia. Neuron. 2002; 36:57-68.

7. Kim SY, Bae JC, Kim JY, et al. Activation of p38 MAP kinase in the rat dorsal root ganglia and spinal cord following peripheral inflammation and nerve injury. Neuroreport. 2002;13:2483-6.

8. Jin SX, Zhuang ZY, Woolf CJ, Ji RR. p38 mitogen-activated protein kinase is activated after a spinal nerve ligation in spinal cord microglia and dorsal root ganglion neurons and contributes to the generation of neuropathic pain. J Neurosci. 2003;23: 4017-22.

9. Obata K, Yamanaka H, Kobayashi K, et al. Role of mitogenactivated protein kinase activation in injured and intact primary afferent neurons for mechanical and heat hypersensitivity after spinal nerve ligation. J Neurosci. 2004;24:10211-22.

10. Lim EJ, Jeon HJ, Yang GY, et al. Intracisternal administration of mitogen activated protein kinase inhibitors reduced mechanical allodynia following chronic constriction injury of infraorbital nerve in rats. Prog Neuropsychopharmacol Biol Psychiatry. 2007:31:1322-9.

11. Vongs A, Lynn NM, Rosenblum CI. Activation of MAP kinaseby MC4-R through PI3 kinase. Regul Pept. 2004;120:113-18.

12. Daniels D, Patten CS, Roth JD, Yee DK, Fluharty SJ. Melanocortin receptor signaling through mitogen-activated protein kinase in vitro and in rat hypothalamus. Brain Res. 2003;986:1-911.

13. Bennett GJ, Xie YK. A peripheral mononeuropathy in rat that produces disorders of pain sensation like those seen in man. Pain. 1998;33:87-107.

14. Yaksh TL. Chronic catheterisation of the spinal subaraehnoid space. Physiol Behav. 1976;17:1031-6.

15. Mountjoy KG, Mortrud MT, Low MJ, Simerly RB, Cone RD. Localization of the melanocortin-4 receptor (MC4-R) in neuroendocrine and autonomic control circuits in the brain. Mol Endocrino. 1994;1 8:1298-308.

16. Kraan M, Tatro JB, Entwistle ML, et al. Expression of melanocortin receptors and pro-opiomelanocortin in the rat spinal cord in relation to neurotrophic effects of melanocortins. Brain Res Mol Brain Res.1999;63:276-86.
17. Kishi T, Aschkenasi CJ, Lee CE, Mountjoy KG, Saper CB, Elmquist JK. Expression of melanocortin 4 receptor mRNA in the central nervous system of the rat. J Comp Neurol. 2003;457: 213-35.

18. Spencer JD, Schallreuter KU. Regulation of pigmentation in human epidermal melanocytes by functional high affinity- $\beta$ melanocyte-stimulating hormone/melanocortin-4 receptor signaling. Endocrinology. 2009;150:1250-8.

19. Tao YX. The melanocortin-4 receptor: physiology, pharmacology, and pathophysiology. Endocr Rev. 2010; 31(4):1-38.

20. Selkirk JV, Nottebaum LM, Lee J, Yang W, Foster AC, Lechner SM. Identification of differential melanocortin 4 receptor agonist profiles at natively expressed receptors in rat cortical astrocytes and recombinantly expressed receptors in human embryonic kidney cells. Neuropharmacology. 2007;52:459-66.

21. Caruso C, Durand D, Schioth HB, Rey R, Seilicovich A, Lasaga M M. Activation of melanocortin 4 receptors reduces the inflammatory response and prevents apoptosis induced by lipopolysaccharide and interferon in astrocytes. Endocrinology. 2009;148:4918-26.

22. Kalange AS, Kokare DM, Singru PS, Upadhya MA, Chopde CT, Subhedar NK. Central administration of selective melanocortin 4 receptor antagonist HS014 prevents morphine tolerance and withdrawal hyperalgesia. Brain Res. 2007;1181:10-20.

23. Vrinten $\mathrm{DH}$, Adan $\mathrm{RA}, \mathrm{Groen} \mathrm{Gj}$, et al. Chronic blockade of melanocotin receptors alleviates allodynia in rats with neuropathic pain. Anesth Analg. 2001;93:1572-7.

24. Bertorelli R, Fredduzzi S, Tarozzo G, et al. Endogenous and exogenous melanocortin antagonists induce antiallodynic effects in a model of rat neuropathic pain. Behav Brain Res. 2005;157: $55-62$.

25. Schafers M, Svensson C.I, Sommer C, Sorkin LS. Tumor necrosis factor-alpha induces mechanical allodynia after spinal nerve ligation by activation of p38 MAPK in primary sensory neurons. J Neurosci. 2003;23:2517-21.

26. Xu L, Huang YG. The influence of p38 mitogen-activated protein kinase inhibitor on synthesis of inflammatory cytokine tumor necrosis factor Alpha in spinal cord of rats with chronic constriction injury. Int Anesth Res Soc. 2007;105(6):1838-44.

27. Zhang FE, Cao JL. Activation of p38 mitogen-activated protein kinase in spinal cord contributes to chronic constriction injuryinduced neuropathic pain. Acta Physiologica Sinica. 2005;57(5): 545-51.

28. Lee KM, Jeon SM, Cho HJ. Interleukin-6 induces microglial CX3CR1 expression in the spinal cord after peripheral nerve injury through the activation of p38 MAPK. Euro J Pain. 2010; 14(7):682.e1-12.

29. Wen YR, Suter MR, Kawasaki Y, et al. Nerve conduction blockade in the sciatic nerve prevents but does not reverse the activation of p38 mitogen-activated protein kinase in spinal microglia in the rat spared nerve injury model. Anesthesiology. 2007;107: 312-21.

30. Chai BX, Li JY. Melanocortin-4 receptor-mediated inhibition of apoptosis in immortalized hypothalamic neurons via mitogenactivated protein kinase. Peptides. 2006;27:2846-57.

31. Chu HC, LuoYJ, Sun XP. Role of melanocortin receptor 4 in excitatory amino acid release from rat astrocytes in spinal cord. Chinese J Anesth. 2010;30(5):569-74. 